\title{
MEchatronic REspiratory System SImulator for Neonatal Applications (MERESSINA) project: a novel bioengineering goal
}

HYPOTHESIS

Rosa T Scaramuzzo ${ }^{1,2}$
Massimiliano Ciantelli'
Ilaria Baldoli
Lisa Bellanti'
Marzia Gentile'
Francesca Cecchi ${ }^{3}$
Emilio Sigali'
Selene Tognarelli ${ }^{3}$
Paolo Ghirri'-4
Stefano Mazzoleni
Arianna Menciassi
Armando Cuttano'
Antonio Boldrini'-4
Cecilia Laschi ${ }^{3}$
Paolo Dario
'Centro di Formazione e Simulazione
Neonatale "NINA," UO Neonatologia,
Azienda Ospedaliera Universitaria
Pisana, Pisa, Italy; ${ }^{2}$ Istituto di Scienze
della Vita, ${ }^{3}$ The BioRobotics Institute,
Scuola Superiore Sant'Anna, Pisa, Italy;
${ }^{4}$ Università di Pisa, Pisa, Italy

Correspondence: Armando Cuttano UO Neonatologia, Azienda Ospedaliera Universitaria Pisana, Via Roma, 67 - 56100 Pisa, Italy Tel +393387764400 Fax +39050992611 Email acuttano@gmail.com
This article was published in the following Dove Press journal:

Medical Devices: Evidence and Research

7 August 2013

Number of times this article has been viewed
Abstract: Respiratory function is mandatory for extrauterine life, but is sometimes impaired in newborns due to prematurity, congenital malformations, or acquired pathologies. Mechanical ventilation is standard care, but long-term complications, such as bronchopulmonary dysplasia, are still largely reported. Therefore, continuous medical education is mandatory to correctly manage devices for assistance. Commercially available breathing function simulators are rarely suitable for the anatomical and physiological realities. The aim of this study is to develop a high-fidelity mechatronic simulator of neonatal airways and lungs for staff training and mechanical ventilator testing. The project is divided into three different phases: (1) a review study on respiratory physiology and pathophysiology and on already available single and multicompartment models; (2) the prototyping phase; and (3) the on-field system validation.

Keywords: simulation, lung, newborn, continuous medical education, respiratory system

\section{Background}

\section{Neonatal lung care: the medical background}

Respiratory function is a fundamental topic in neonatology and essential in enabling birth to occur. In fact, moving from the uterus to the external postnatal environment requires a correctly functioning heart and lungs since the placenta no longer provides cardiopulmonary functions.

Since the 1970s, neonatologists have been able to use drugs (eg, surfactants, caffeine, etc) and advanced devices for mechanical ventilation. Indeed, invasive ventilation via endotracheal tube is one of the most common therapeutic interventions performed in infants with respiratory failure. Respiratory distress syndrome occurs in about $50 \%$ of preterm infants born at less than 30 weeks of gestational age. Mechanical ventilation using conventional or high-frequency ventilation and surfactant therapy has become the standard care in management of preterm infants affected by respiratory distress syndrome. ${ }^{1}$

Respiratory assistance in newborns is challenging due to high interpatient variability: weight varies from 400 grams to a few kilos and lung status is strictly related to gestational age. In fact, at 24 weeks of gestational age, a preterm newborn's lung includes only sacculi, whereas at term of pregnancy ( 40 weeks), there are millions of alveoli able to increase in number even after birth (to a whole air/blood exchange surface of 70-100 $\left.\mathrm{m}^{2}\right){ }^{2}$ Congenital abnormalities of lung, heart, or respiratory muscles, as well as acquired perinatal diseases, such as pneumonia or meconium aspiration syndrome, may eventually complicate the scene. Moreover, the same mechanical 
ventilation equipment is used on patients affected by different pathologies and disorders.

In 1967, Northway ${ }^{3}$ described a group of newborns suffering from bronchopulmonary dysplasia (BPD), which is related to lung damage due to mechanical ventilation and oxygen supply. After that original paper, it was demonstrated that mechanical ventilation is able to cause side effects in other organs (ie, arteries, brain, heart, gut, eye) as well, even if, at present, it is well known that barotrauma and volutrauma resulting from mechanical ventilation are cofactors in the pathogenesis of BPD - a multifactorial process linked to immature lung tissue, oxidant injury, and proinflammatory mediators. ${ }^{4}$ BPD represents the most common severe complication in preterm infants: birth weight and gestational age are main predictors of this disease..$^{5}$ It can be considered one of the most severe complications of mechanical ventilation in survivors. Among neonates, about 35\% with a birth weight between 500 and 750 grams and $25 \%$ with a birth weight between 750 and 1000 grams develop chronic lung disease, a complication that predisposes to tracheo- and bronchomalacia, recurrent infections, and pulmonary hypertension with high mortality $(25 \%-30 \%)$ and poor quality of life.

Reduction of the incidence and severity of BPD may be possible through a reduction of the amount of injury induced by respiratory support interventions. ${ }^{4}$ Current management of the disease aims at maintaining an adequate gas exchange while promoting optimal lung growth. ${ }^{6}$ During hospitalization, the respiratory goals should aim to provide adequate oxygenation, permissive hypercapnia, and gentle ventilation. ${ }^{7}$ However, oxygen saturation and gas blood values, commonly monitored in clinical practice, provide only partial and vicarious assessment of ventilation efficacy. ${ }^{8,9}$ The same applies to pressure or flow controlled system of ventilators, the application point of which are located far from newborn trachea or alveoli.

Given the abovementioned issues, specific training of physicians and nurses on newborn ventilation is crucial for optimal outcomes.

\section{Simulation-based training}

Use of simulation-based training is quickly becoming a source of experiential learning in medical education. This method was proposed in USA about a decade ago, and more recently in Europe. It provides safe and effective procedures for the practice and acquisition of clinical skills needed for patient care. Nowadays, the inclusion of such medical education is aimed at improving the abilities of health care professionals to ensure patient safety. To date, the use of simulation is a common tool in undergraduate and graduate medical education, whereas its utilization in continuing medical education (CME) has yet to be explored. Currently, CME training programs are increasingly focusing on simulation to optimize learning outcomes..$^{10,11}$

\section{Previous lung simulators: the bioengineering background}

To date, most common systems for medical training are passive simulators consisting of tubes, variable resistances, and air sacs with expandable volumes. Examples of such systems include the Adult/Infant Training and Test Lung (Michigan Instruments, Grand Rapids, MI, USA), the ACCU LUNG Precision Test Lung (Fluke Biomedical, Everett, WA, USA), and the SmartLung ${ }^{\mathrm{TM}}$ (imtmedical, Buchs, Switzerland). Such devices are small and portable and allow the operator to set airway resistance, pulmonary compliances, and volumes, but are unable to provide autonomous breathing patterns, and cannot be used to evaluate the dynamic interaction with mechanical ventilators.

On the other hand, currently available pulmonary active simulators often allow simulation of only a limited set of clinical situations, due to their architecture being based on single- or double-compartment models. These structures do not provide a realistic representation of physiological and pathological newborn conditions (eg, times of apnea, localized edemas or barotraumas, obstructions of specific portions of the airway tree, etc). Usually they consist of rigid containers with plungers or compliant rubber bellows with springs, and are too bulky to be inserted into physiological human phantoms.

The IngMar Adult/Pediatric Lung Model $^{\circledR}$ and ASL 5000 Adult/Neonatal Breathing Simulator ${ }^{\circledR}$ (IngMar Medical, Ltd, Pittsburgh, PA, USA) represent examples of devices capable of replicating parts of the pulmonary mechanics of healthy and pathological infants. With these devices, the respiratory pattern can be set and mechanical parameters characterizing the pulmonary system can be continuously controlled. Lungs are sketched as composed of two homogeneous compartments, without any differentiation in terms of compliance and resistance.

In addition, currently available realistic infant puppetlike simulators, such as the $\operatorname{SimNewB}{ }^{\circledR}$ (Laerdal Medical, Wappingers Falls, NY, USA) and the Newborn HAL ${ }^{\circledR}$ S3010 (Gaumard, Miami, FL, USA), are able to replicate several vital functions and active breathing; oral/nasal intubation can be performed as well. They also allow the operator to control respiration rate and depth and to observe chest-rise during breathing. However, such simulators, on which only single resistance and compliance values can be set, are not able to 
represent complex breathing patterns and do not allow for the evaluation of specific pathological alterations.

\section{Study aim}

The main objective of the MEchatronic REspiratory System SImulator for Neonatal Applications (MERESSINA) project is to design and develop a mechanical system able to replicate the respiratory act of a newborn. Microsystem technologies are considered and integrated into a functional system for clinical applications. The high-fidelity anatomical and functional simulator of human airways and lungs will be a reliable device with which to train neonatologists, anesthetists, and nurses in neonatal intensive care units (NICUs). It will represent an innovative tool for medical training of mechanical ventilation. The secondary aim is to evaluate the proposed simulator as an effective instrument with which to test mechanical ventilators for improving respiratory assistance in NICUs.

\section{Methods Study design}

The project, lasting 18 months, is divided into three different phases: (1) a theoretical study; (2) the prototyping phase; and

(3) the on-field system validation.

In the first phase (months 1-8), researchers will work simultaneously on the study of respiratory physiology, focusing on research and preliminary analysis of the critical parameters for the simulator design (task 1.1), and on the study of singleand multi-compartment models of the human respiratory system and proper materials for the simulator (task 1.2).

The second phase (months 5-14) will be devoted to the design and development of the several parts composing the system (task 2.1), essentially an electronic unit and a mechanical unit. Moreover, dedicated software for the electronic unit's control will be created (task 2.2). At the same time, clinical protocols to validate the system will be written (task 2.3). This phase of the project begins before the end of the first phase and immediately after the preliminary investigation about models and materials, in order to ensure sufficient time for exhaustive exploration of different solutions.

In the third phase (months 15-18), the system will be tested for on-field validation through high-fidelity training courses for specialized medical staff (task 3.1). On the basis of these courses, important feedback will be gathered in order to optimize the final system (task 3.2) (Figure 1). The simulator will also be validated by means of connection to mechanical ventilators.

\section{Detailed description of all interventions and expected results}

The starting theoretical study will focus on respiratory physiology.

Tissue oxygenation, the main goal of respiration, starts from alveolus ventilation. This process is permitted by thorax expansion. The lung and thorax are in balance due to the lung's elastic retraction force (inside oriented) versus the thorax's elastic traction (outside oriented). During inspiration, the diaphragm tenses and causes an intrapleural pressure reduction, which produces passive alveolus enlargement. Thus, air enters into lungs as long as alveolus pressure is in balance with atmospheric pressure. Newborns, especially if preterm, may experience difficulties in respiration as: (1) lung elasticity (compliance) is decreased, due to low surfactant production and excess in intra-alveolar fluid; (2) thorax compliance is

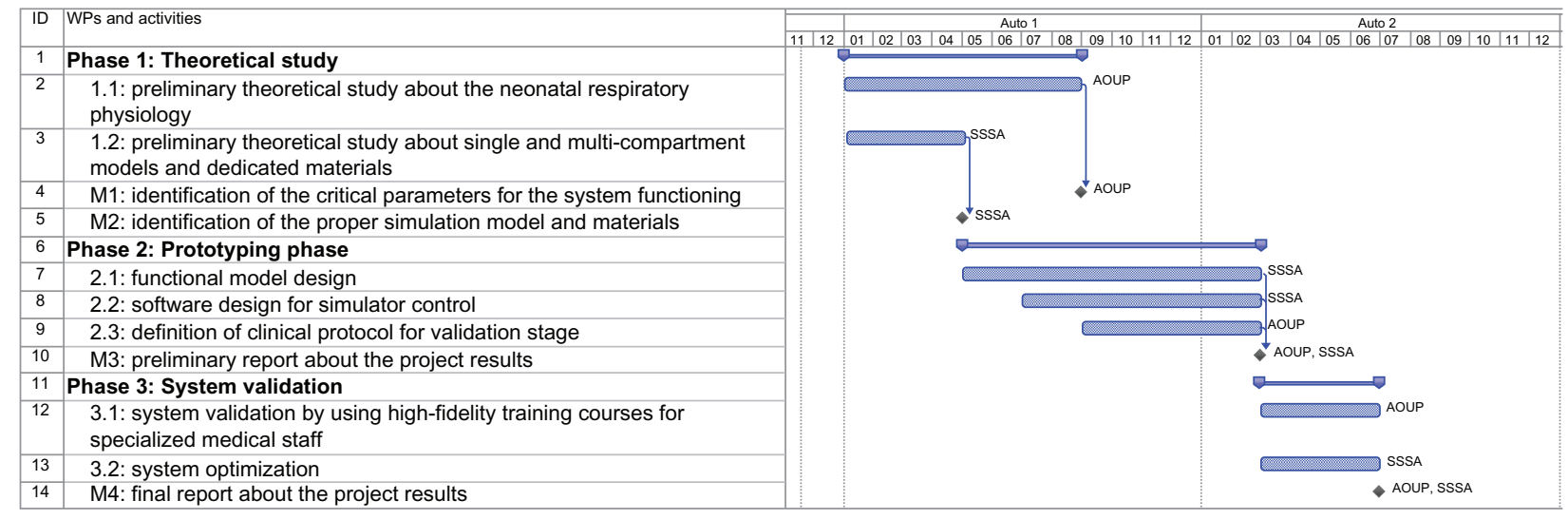

Figure I Gantt diagram.

Note: The scheme illustrates the work plan. The names of the clinical (AOUP) and engineering (SSSA) groups at the end of the activity timeline indicate the groups responsible for each activity.

Abbreviations: WP, work package; SSSA, The BioRobotics Institute, Scuola Superiore Sant'Anna; AOUP, Hospital of Pisa. 
increased; (3) airway resistance is increased. ${ }^{2}$ Moreover, congenital abnormalities of the face, neck, airways, or lungs themselves may complicate the functioning. Finally, preterm birth is associated with immature nervous respiratory control. ${ }^{12}$

As regards the compartmental-model reproduction of the respiratory system, previous studies confirm that, even though each of the millions of alveoli could act as a separate mechanical unit, the mechanics of normal or pathological lungs during quiet breathing can be represented by a singleor double-compartment model. ${ }^{13,14}$ For this reason, the first months of the project will be devoted to a detailed analysis of models reported in literature in order to identify the most appropriate one for our simulator development.

In particular, the multi-compartment model, having five cameras (one for each of the lobes in normal human lungs, ie, two lobes in the left lung plus three lobes in the right lung), is expected to be the most appropriate to develop a mechatronic system able to accurately reproduce respiratory acts.

A first prototype will likely be developed by a plastic tube piston system. Thereafter, state-of-the-art microsystems and smart materials technologies will be investigated in order to select the most suitable solution for our purposes. Smart materials and novel physical processes will be analyzed in order to design a miniaturized system prototype able to show the desired performances by exploiting the intrinsic characteristics of such materials. A variable stiffness system could be obtained by using electroactive polymers or actuators based on granular jamming. So, different materials solutions will be tested from benchmarks of technology to select the most appropriate design for MERESSINA.

At the end of the first phase, two important milestones will be achieved: the identification of the critical parameters of the system (milestone 1) and the choice of model and materials for the development of the simulator (milestone 2).
The second phase will be focused on prototype development. The simulator should be formed of two parts: an electronic unit and a mechanical unit (Figure 2).

The electronic unit will be composed of an electronic board and a personal computer able to control the mechanical unit. The software will be specifically designed to allow the operator to set the different parameters that characterize a specific respiratory pattern and to simulate the critical clinical situations through an easy-to-use graphical user interface.

The mechanical unit will be composed of the lung simulator (air chamber, springs, resistors, etc) embedded in a phantom in order to provide both anatomical and functional representation.

The respiratory system simulator is the key element of our project and will be designed on the basis of multicompartment models identified during the first phase and developed using specific materials (eg silicone, used alone or joined together with smart materials).

A crucial element of the development phase is to provide the system with actuation and sensors in order to replicate high-fidelity characteristics of passive and active lung behavior.

Sensors embedded in the system will provide data about important parameters such as pressure distribution inside the lung, tidal volume, and air flow in the different areas of the lung.

Passive simulators are usually employed for testing the performances of the ventilators through endurance tests, are based on mechanical systems composed of tubes manufactured with standard dimensions and rigid volumes.

Indeed, commercially available active simulators generally use compliant rubber bellows, often equipped with springs, or rigid containers to simulate pulmonary compliances, even if showing some important limitations due to: (1) inability

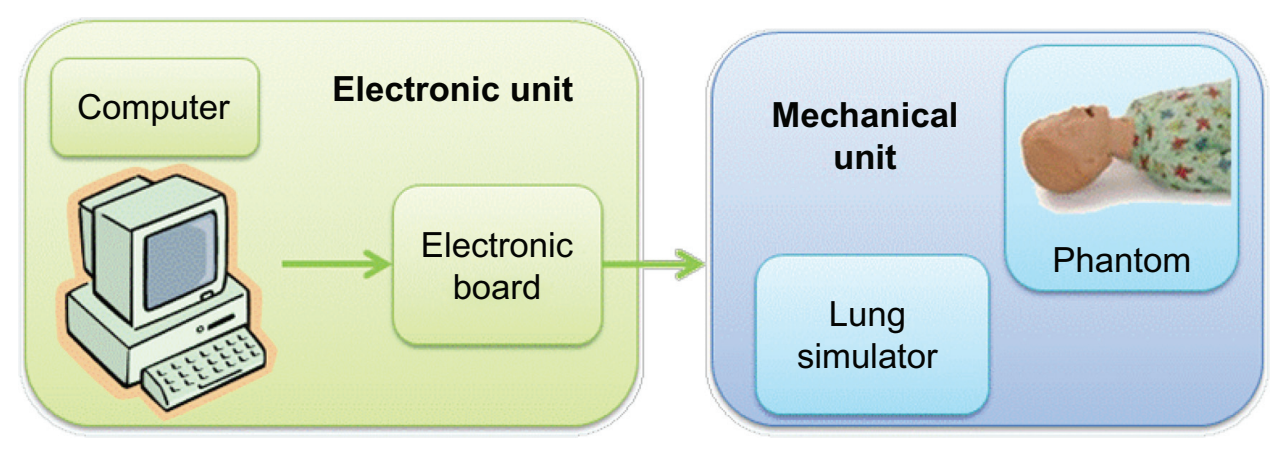

Figure 2 Simulator structure.

Notes: The final simulator will include two parts: an electronic unit (controlled by dedicated software) and a mechanical unit (which could be included in a phantom for medical training). 
to simulate low compliance; (2) bulky dimensions; and (3) limitations to possible simulation scenarios.

The proposed system, through the use of specific sensors and actuators, will be used as a training device through simulations and as an innovative and reliable assessment device for mechanical ventilators.

This system, manufactured to accurately and reliably reproduce preterm respiratory mechanics, will be interfaced to commercial ventilators in order to monitor respiratory parameters in different areas of the lung and for reevaluation of the ventilatory settings (Figure 3).

Specific clinical protocols will be proposed for validation of the mechatronic system. In particular, we will address the most challenging and frequent clinical situations in caregiver education in order to develop and implement an algorithm for diagnosis and treatment.

Figure 4 summarizes the technical features of the project. By way of a flowchart, the system design, assembly, and validation phases are highlighted and reported in order of appearance in the project timeline.

\section{The study setting}

The development of a final prototype able to simulate the complex physiological characteristics of the respiratory system and the main pathological conditions will be achieved through a close and continuous collaboration between bioengineers and medical staff. The prototype design and the development phase will be carried out at the BioRobotics Institute, Scuola Superiore Sant'Anna Pisa, Italy, and the testing and validation procedures will be performed in the NICU at the Pisa University Hospital, Pisa, Italy, which offers the ideal structured environment for the validation process.

The bioengineering tasks will also be carried out at the BioRobotics Institute in Pisa. This is a public university for research and advanced undergraduate and graduate education in the fields of social and experimental sciences (see www. sssup.it). Facilities at the BioRobotics Institute include:

- ultraprecision machining (high-precision CNC [Computer Numerical Control] machining, microwire and microsink electro-discharge machining, focused ion beam, injection molding, microstereolithography and precision electrodeposition, and silicon bulk micromachining);

- clean room (equipment for lithography, silicon micromachining, thin-film deposition [eg sputtering and ion beam evaporator and monomolecular], and monomolecular layer deposition);

- measurement and testing machines; profilometer and ellipsometer; microscopes (atomic force microscope, scanning tunneling microscope, optical fiber microscope, optical microscope); MEMS [Micro Electro-Mechanical Systems] characterization equipment (micromechanical test bench with precision load cell); surface characterization equipment; electrical characterization equipment; optical sensors research equipment (helium-neon laser, UV visible power meter);

- wet lab for in vitro testing and surgical procedures assessment; electromagnetic sensors (NDI, Waterloo, Ontario, Canada); robotic positioning arms;

- cell culture lab (with incubator, microscope, autoclave, vortex, magnetic plate, and ultrasonic bath); and

- simulation and design software (Ansys, Canonsburg, Pennsylvania, USA, Pro-Engineer, PTC, USA).

The simulator will be tested and validated during advanced training courses for specialized staff within the framework of the Education and Simulation Centre for Neonatal Resuscitation "NINA," unique in Italy. ${ }^{15}$

The "NINA" Centre (see www.simulazionenina.it; www. neonatologia.unipi.it) participates in the project through use of staff knowledge on respiratory diseases in newborns and on their skills in neonatal intensive care. Moreover, medical

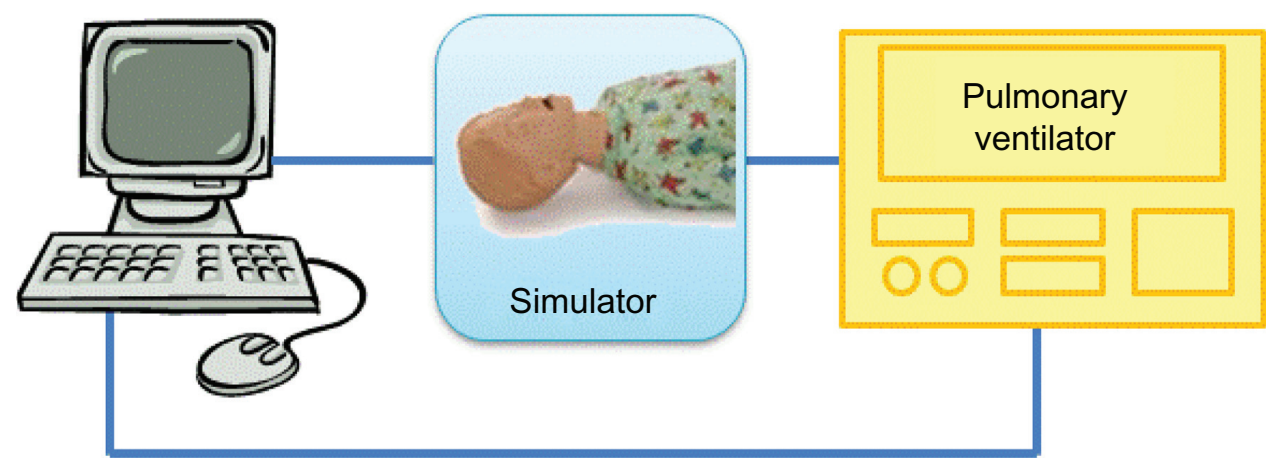

Figure 3 A potential simulator scenario.

Note: The final simulator will represent a reliable tool with which to test mechanical ventilators for newborns. 


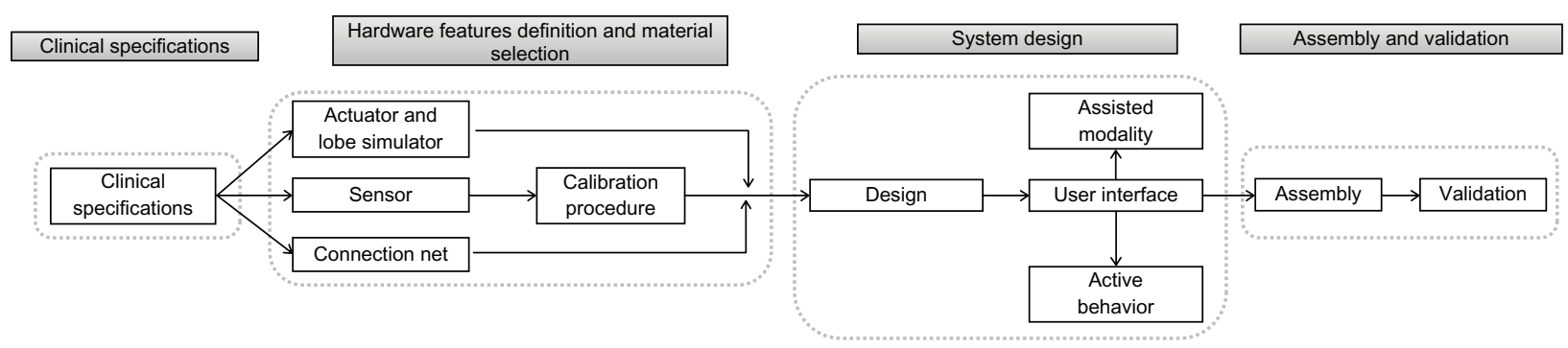

Figure 4 Project flowchart.

staff and specific equipment will be used during the training courses to test and validate the proposed simulator (third period of the project).

The "NINA" Centre for Neonatal Resuscitation is located in the NICU at Pisa University Hospital, according to the "justin-time, just-in-place" rule of high-fidelity education. ${ }^{14}$

\section{Discussion}

Nowadays, biomedical engineering, characterized by integration of information technology, electronics, mechanics, chemistry, and material science, has a leading role in the emerging field of medical education and training, as it can strongly contribute to the design and development of innovative technological devices able to simplify and improve clinical learning activities.

The close collaboration between medical staff and bioengineers represents a key factor for developing mechatronic devices replicating the complex nature of physiological systems, in terms of architecture and active behavior. Moreover, an ideal simulation system should have an anatomical resemblance for emphasizing the involvement of the operator during training.

Unfortunately, to date, commercially available simulators of breathing function rarely adhere to real anatomy and physiology of the respiratory system, especially in the case of neonatal representation.

The relevance of the MERESSINA project is based on a novel approach to respiratory pathophysiology.

Barotrauma and volutrauma as secondary side effects of mechanical ventilation represent two of the main pathogenetic factors responsible for chronic lung disease. Efforts of medical and paramedical staff are aimed at minimizing lung injury secondary to mechanical ventilation, improving the quality of life of vulnerable patients, and reducing their risk of further damage. In this scenario, it is essential to optimize the clinical support, relying on simulation systems for medical training.

The development of a respiratory system simulator is challenging. Mechanisms underlying gas-blood exchange in vivo are several (mechanical, chemical, temperature dependent) and extremely complex. Currently available models, which are used to check air pumping in ventilators, are based on a simplified (single- or bicompartmental) scheme. The need for simulating complex elastic and resistance lung characteristics represents a challenging task, and this may realistically explain the abovementioned simplification.

An electronic control system able to reproduce respiration variability, apneas, cough, hiccups, sobbing, and bronchoconstriction is also needed. A graphical user interface is necessary for the establishment and analysis of respiratory models.

The MERESSINA project exploits current knowledge to propose an innovative approach to be used in CME training courses. Medical and paramedical staff will interact with a dummy that reproduces a neonate, representing the highfidelity system.

Specific high-fidelity training courses will be arranged through specific interventions and ventilation strategies. High-fidelity simulation implies a completely interactive training system based on innovative teaching strategies in a realistic clinical scenario. Physicians and nurses must use devices and understand patient-device interactions. For such purpose, a realistic simulator provides the operator with high involvement and easier learning procedures. Operators can ultimately be certified as having expertise in the field of newborn ventilation. In addition, such a simulator could be used for the assessment of mechanical ventilators in NICUs as well.

\section{Conclusion}

The proposed neonatal respiratory simulator could represent a reliable tool for medical staff education, while keeping patients safe, and for industries, to optimize mechanical ventilators. A patent represents a potential outcome of the presented research project.

\section{Research status}

At the time of manuscript submission (March 2013), the first phase of the research (theoretical study and literature review) was completed. We are currently working on the second phase (prototype design). 


\section{Author contributions and information}

$\mathrm{AC}$, chief investigator and coordinator for the medical section, conceived the study, participated in the design, and helped to draft the manuscript. PD, AM, and CL coordinated the engineering section; and together with SM are responsible for the prototype and testing phase, as well as revising the manuscript. RTS, MC, MG, FC, and ST participated in the study design and are responsible for data collection and draft coordination/writing. MC, MG, and RTS were responsible for research on physiology and pathophysiology of the newborn respiratory system. $\mathrm{ES}, \mathrm{MC}, \mathrm{AC}$, and $\mathrm{AB}$ were responsible for model testing of mechanical ventilators and simulation training sessions. IB and LB were responsible for materials and software development. Moreover, they contributed to the manuscript drafting and critically reviewed it. PG, ES, and $\mathrm{AB}$ reviewed the study and this manuscript. FC and ST created the figures for this manuscript. All authors contributed to the development of the protocol and read and approved the final manuscript.

AC, AB, PG, ES, MC, MG, and RTS work at Centro di Formazione e Simulazione Neonatale "NINA," located inside the Neonatal Care Unit of Pisa Hospital. The center is a unique structure in Italy, specialized in training by high-fidelity simulation for health care staff working in Neonatology. Therefore, they have been involved in identifying novel strategies for learning, in order to guarantee a high quality of health assistance and patient safety. IB, LB, FC, ST, AM, CL, SM, and PD work at the BioRobotics Institute, Scuola Superiore Sant'Anna, which has expertise in the design and development of surgical, diagnostic, and therapeutic robotic systems and microtechnologies for medical applications.

\section{Acknowledgments}

The authors would like to acknowledge Azienda Ospedaliera Universitaria Pisana (Santa Chiara Hospital, Pisa, Italy) for financial support. The research received major funding in the form of a grant by AGENAS, Commissione Nazionale per la Formazione Continua - Italian Ministry of Health, grant "Sviluppo e ricerca sulle metodologie innovative nella formazi- one continua - anno 2011" (Grant n - Codice gara: 4353869. Approval number for funding - Codice CIG: 4415895FD1).

\section{Disclosure}

The authors report no conflicts of interest in this work.

\section{References}

1. Ramanathan R. Optimal ventilatory strategies and surfactant to protect the preterm lungs. Neonatology. 2008;93(4):302-308.

2. Moretti C, Papoff P. Lung development and pulmonary malformations. In: Buonocore G, Bracci R, Weindling M, editors. Neonatology $-A$ Practical Approach to Neonatal Management. Springer-Verlag, Milan, Italy. Ed; 2012.

3. Northway WH, Rosan RC, Porter DY. Pulmonary disease following respirator therapy of hyaline-membrane disease. Bronchopulmonary dysplasia. N Engl J Med 1967;276(7):357-368.

4. Mosca F, Colnaghi M, Fumagalli M. BPD: old and new problems. J Matern Fetal Neonatal Med. 2011;24 Suppl 1:80-82.

5. Jobe AH. The new bronchopulmonary dysplasia. Curr Opin Pediatr. 2011;23(2):167-172.

6. Papoff P, Cerasaro C, Caresta E, Barbàra CS, Midulla F, Moretti C. Current strategies for treating infants with severe bronchopulmonary dysplasia. J Matern Fetal Neonatal Med. 2012;25 (Suppl 3):15-20.

7. Kugelman A, Durand M. A comprehensive approach to the prevention of bronchopulmonary dysplasia. Pediatr Pulmonol. 2011;46(12): 1153-1165.

8. van der Eijk AC, Horsch S, Eilers PH, Dankelman J, Smit BJ "New-generation" pulse oximeters in extremely low-birth-weight infants: how do they perform in clinical practice? J Perinat Neonatal Nurs. 2012;26(2):172-180.

9. Sivarajan VB, Bohn D. Monitoring of standard hemodynamic parameters: heart rate, systemic blood pressure, atrial pressure, pulse oximetry, and end-tidal CO2. Pediatr Crit Care Med. 2011; 12(Suppl 4):S2-S11.

10. McGaghie WC, Siddall VJ, Mazmanian PE, Myers J; American College of Chest Physicians Health and Science Policy Committee. Lessons for continuing medical education from simulation research in undergraduate and graduate medical education: effectiveness of continuing medical education: American College of Chest Physicians Evidence-Based Educational Guidelines. Chest. 2009;135(Suppl 3):62S-68S.

11. Curtis MT, DiazGranados D, Feldman M. Judicious use of simulation technology in continuing medical education. $J$ Contin Educ Health Prof. 2012;32(4):255-260.

12. Alvaro R, Rigatto H. Control of breathing in newborns. In: Buonocore G, Bracci R, Weindling M, editors. Neonatology - A Practical Approach to Neonatal Management. Springer-Verlag, Milan, Italy. Ed; 2012.

13. Ligas JR, Primiano FP. Encyclopedia of Medical Devices and Instrumentation, Webster JG, editor. New York: Wiley;1988:2550-2573.

14. Cappa P, Sciuto SA, Silvestri S. A novel preterm respiratory mechanics active simulator to test the performances of neonatal pulmonary ventilators. Rev Sci Instrum. 2002;73(6):2411-2416.

15. Cuttano A, Scaramuzzo RT, Gentile M, et al. High-fidelity simulation in neonatology and the experience of Nina. Journal of Pediatric and Neonatal Individualized Medicine (JPNIM). 2012;1(1):67-72.
Medical Devices: Evidence and Research

\section{Publish your work in this journal}

Medical Devices: Evidence and Research is an international, peerreviewed, open access journal that focuses on the evidence, technology, research, and expert opinion supporting the use and application of medical devices in the diagnosis, treatment and management of clinical conditions and physiological processes. The identification of novel

\section{Dovepress}

devices and optimal use of existing devices which will lead to improved clinical outcomes and more effective patient management and safety is a key feature. The manuscript management system is completely online and includes a quick and fair peer-review system. Visit http://www. dovepress.com/testimonials.php to read real quotes from authors. 